\title{
HOMOTOPY THEORY AND CIRCLE ACTIONS ON SYMPLECTIC MANIFOLDS
}

\author{
JOHN OPREA \\ Department of Mathematics, Cleveland State University \\ Cleveland, Ohio 44115, U.S.A. \\ E-mail: oprea@math.csuohio.edu
}

Introduction. Traditionally, soft techniques of algebraic topology have found much use in the world of hard geometry. In recent years, in particular, the subject of symplectic topology (see $[\mathrm{McS}]$ ) has arisen from important and interesting connections between symplectic geometry and algebraic topology. In this paper, we will consider one application of homotopical methods to symplectic geometry. Namely, we shall discuss some aspects of the homotopy theory of circle actions on symplectic manifolds. Because this paper is meant to be accessible to both geometers and topologists, we shall try to review relevant ideas in homotopy theory and symplectic geometry as we go along. We also present some new results (e.g. see Theorem 2.12 and §5) which extend the methods reviewed earlier. This paper then serves two roles: as an exposition and survey of the homotopical approach to symplectic circle actions and as a first step to extending the approach beyond the symplectic world.

1. Review of symplectic geometry. A manifold $M^{2 n}$ is symplectic if it possesses a nondegenerate 2 -form $\omega$ which is closed (i.e. $d \omega=0$ ). The nondegeneracy condition is equivalent to saying that $\omega^{n}$ is a true volume form (i.e. nonzero at each point) on $M$. Furthermore, the nondegeneracy of $\omega$ sets up an isomorphism between 1-forms and vector fields on $M$ by assigning to a vector field $X$ the 1-form $i_{X} \omega=\omega(X,-)$. The symplectic manifold $M$ is then denoted by $\left(M^{2 n}, \omega\right)$. For the basic theory of symplectic manifolds, see $[\mathrm{Au} 1],[\mathrm{AM}],[\mathrm{AL}],[\mathrm{ABKLR}]$ and $[\mathrm{McS}]$ for instance.

1991 Mathematics Subject Classification: 57S99, 55P99, 58F99.

This paper, which is in final form and no version of which will be published elsewhere, is based on a series of lectures given at the Conference on Homotopy and Geometry, June 9-13, 1997 held at the Stefan Banach Center in Warsaw, Poland. I wish to thank the Center for its hospitality during my stay in Poland. 
ExAmples 1.1. (1) Let $\mathbb{R}^{2 k}$ denote Euclidean space with coordinates $\left\langle x^{1}, \ldots, x^{k}\right.$, $\left.y^{1}, \ldots, y^{k}\right\rangle$. It is then easy to see that the form $\omega=\sum_{i=1}^{k} d x^{i} \wedge d y^{i}$ is symplectic by observing that $\omega^{k}$ is a volume form.

(2) For any manifold $M^{k}$, the cotangent bundle $T^{*} M$ is a symplectic manifold with a canonical symplectic form defined locally by a 1-form $\theta=\sum_{i=1}^{k} p_{i} d q^{i}$ with exact symplectic 2 -form

$$
\omega=-d \theta=\sum_{i=1}^{k} d q^{i} \wedge d p_{i} .
$$

Here, $\left(q^{1}, \ldots, q^{k}, p_{1}, \ldots, p_{k}\right)$ are local coordinates, the $q^{i}$ 's coming from $M$ and the $p_{j}$ 's coming from $T^{*} M$.

(3) In fact, the first two examples will not be of interest to us. From now on, we will consider only closed (i.e. compact without boundary) manifolds. From (1), we can obtain an example, however, by noting that the symplectic form on $\mathbb{R}^{2 k}$ is invariant under translations $x^{i} \mapsto x^{i}+2 \pi$ and $y^{i} \mapsto y^{i}+2 \pi$, so this form induces a symplectic form on the orbit space $T^{2 k}=\mathbb{R}^{2 k} / \mathbb{Z}^{2 k}$. Hence, the even tori have natural symplectic structures derived from Euclidean space.

(4) Perhaps the most important example of symplectic manifolds from the viewpoint of geometry are Kähler manifolds. Let $M$ denote a complex $k$-manifold endowed with a Hermitian metric (in local analytic coordinates $\left(z^{1}, \ldots, z^{k}\right)$

$$
h=\sum_{i=1}^{k} h_{i j} d z d \bar{z} .
$$

Writing $d z^{i}=d x^{i}+i d y^{i}$ and $d \bar{z}^{j}=d x^{j}-i d y^{j}$, we can expand the expression above into its real and imaginary parts to get

$$
h=g+i \omega
$$

where $g$ is a Riemannian metric on $M$ and $\omega$ is a 2-form (which must be nondegenerate since it is the alternating part of a Hermitian form). The 2 -form $\omega$ may be written as

$$
\omega=-\frac{i}{2} \sum_{i=1}^{k} h_{i j} d z^{i} \wedge d \bar{z}^{j} .
$$

Then, if $\omega$ is closed, $M$ is said to be a Kähler manifold. By what we have said above, all Kähler manifolds are symplectic. Examples of Kähler manifolds include $\mathbb{C} P^{k}$ for all $k$ as well as all smooth projective varieties $V$. In this case, the 2 -form $\omega$ is obtained as the pullback of the Kähler form of $\mathbb{C} P^{k}$ via the inclusion $V \hookrightarrow \mathbb{C} P^{k}$. A compact Kähler manifold $M^{2 k}$ also has the Hard Lefschetz Property which says (in part) that multiplication by powers of the Kähler cohomology class, also denoted by $\omega$, induce cohomology isomorphisms

$$
\omega^{j}: H^{k-j}(M ; \mathbb{Q}) \stackrel{\cong}{\longrightarrow} H^{k+j}(M ; \mathbb{Q}) .
$$

Furthermore, because forms on a Kähler manifold decompose into holomorphic and antiholomorphic parts, there is a Hodge decomposition on cohomology with

$$
H^{p, q}(M ; \mathbb{C}) \cong \bar{H}^{q, p}(M ; \mathbb{C})
$$


so that $H^{n}(M ; \mathbb{C})=\oplus_{p+q=n} H^{p, q}(M ; \mathbb{C})$. The isomorphism above then shows that, since odd numbers have symmetric partitions into two numbers, in odd degrees, Betti numbers must be even. In even degrees, because $\omega^{j} \neq 0$, the cohomology of a compact Kähler manifold is always nonzero.

(5) If $G$ is a compact Lie group, then it acts on the dual of its Lie algebra $\mathfrak{g}^{*}$ by the coadjoint action. It is a fact that the orbits of this action are always symplectic manifolds. For instance, the coadjoint action of $S O(3)$ on $s o(3) \cong \mathbb{R}^{3}$ actually corresponds to rotation in $\mathbb{R}^{3}$, so the orbits of the action are 2 -spheres of varying radii. Of course, 2 -spheres (as well as all oriented surfaces) are symplectic.

The fundamental theorem about symplectic manifolds is a generalization of the situation for cotangent bundles.

TheOREM 1.2 (Darboux's Theorem). Around each point in a symplectic manifold $\left(M^{2 k}, \omega\right)$ there are local coordinates $\left(x^{1}, \ldots, x^{k}, y^{1}, \ldots, y^{k}\right)$ such that

$$
\omega=\sum_{i=1}^{k} d x^{i} \wedge d y^{i} .
$$

This result says that symplectic manifolds have no local distinguishing invariants. In this sense, symplectic geometry is a global subject.

EXAMPLE 1.3. Symplectic geometry is the natural framework for Hamiltonian mechanics. Given a smooth function $H: M \rightarrow \mathbb{R}$ (i.e. the Hamiltonian), let $X_{H}$ be the vector field on $M$ determined by the isomorphism between 1-forms and vector fields,

$$
i_{X_{H}} \omega=d H .
$$

Take Darboux coordinates $\left(q^{i}, p_{i}\right)$ so that $\omega=\sum d q^{i} \wedge d p_{i}$ and

$$
X_{H}=\sum \dot{q}^{i} \frac{\partial}{\partial q^{i}}+\sum \dot{p}_{j} \frac{\partial}{\partial p_{j}}
$$

where $\dot{q}^{i}$ and $\dot{p}_{j}$ are tangent vectors to the integral curves of $X_{H}$. Now, the forms of $\omega$ and $X_{H}$ immediately give

$$
\dot{q}^{i}=\omega\left(X_{H}, \frac{\partial}{\partial p_{i}}\right)=d H\left(\frac{\partial}{\partial p_{i}}\right)=\frac{\partial H}{\partial p_{i}}
$$

and

$$
\dot{p}_{i}=-\omega\left(X_{H}, \frac{\partial}{\partial q^{i}}\right)=-d H\left(\frac{\partial}{\partial q^{i}}\right)=-\frac{\partial H}{\partial q^{i}} .
$$

These are Hamilton's equations of course, so the symplectic form is seen as providing the proper theoretical avenue for studying mechanics.

The example above provides the motivation for studying $S^{1}$ actions on symplectic manifolds. The circle $S^{1}$ acts symplectically on the symplectic manifold $\left(M^{2 n}, \omega\right)$ if each $g \in S^{1}$ satisfies $g^{*} \omega=\omega$ as forms. We denote the action by $A: S^{1} \times M \rightarrow M$ and the orbit map at $m$ by $a_{m}(g)=A(g, m)$ (or simply $a$ when no confusion can arise). The orbit map induces a map of tangent spaces $T_{e} a: T_{e} S^{1} \rightarrow T_{m} M$ for any given $m$, so by fixing a unit vector $X$ in $T_{e} S^{1} \cong \mathbb{R}$ and mapping by $T_{e} a$ we obtain a fundamental vector field $\underline{X}$ on $M$. Recall that the interior multiplication operator $i_{X}$ is defined on a 
$k$-form $\theta$ by $i_{\underline{X}} \theta\left(Y_{1}, \ldots, Y_{k-1}\right)=\theta\left(\underline{X}, Y_{1}, \ldots, Y_{k-1}\right)$. The operator $i_{\underline{X}}$ is a derivation on the commutative differential graded algebra of de Rham forms and it satisfies

$$
L_{\underline{X}}=i_{\underline{X}} d+d i_{\underline{X}},
$$

where $L_{\underline{X}}$ is the Lie derivative and $d$ is exterior differentiation. The definition of $L_{\underline{X}}$ and the $S^{1}$-invariance of the closed form $\omega$ imply that

$$
0=L_{\underline{X}}(\omega)=d i_{\underline{X}} \omega .
$$

Hence, $i_{X} \omega$ is a closed 1-form. The $S^{1}$-action is said to be Hamiltonian if $i_{X} \omega$ is exact. That is, the action is Hamiltonian if there is a smooth function $\mu: M \rightarrow \mathbb{R}$ with $d \mu=i_{\underline{X}} \omega$. This description may, of course, be generalized to torus actions as well.

Now, let $H: M \rightarrow \mathbb{R}$ be a Hamiltonian and define a Hamiltonian vector field $X_{H}$ by $i_{X_{H}} \omega=d H$. The integral curves (i.e. the motions of the system) of $X_{H}$ satisfy Hamilton's equations

$$
\dot{q}^{i}=\frac{\partial H}{\partial p^{i}} \quad \dot{p}^{i}=-\frac{\partial H}{\partial q^{i}}
$$

where $q^{i}, p^{i}$ are coordinates for which $\omega=\sum d q^{i} \wedge d p_{i}$ (by Darboux's theorem). Suppose $A: S^{1} \times M \rightarrow M$ is a Hamiltonian circle action with $i_{X} \omega=d f$. Recall that the Poisson bracket of functions $H$ and $f$ is defined to be

$$
\{f, H\}=\omega\left(\underline{X}, X_{H}\right) .
$$

The circle $S^{1}$ acts as a Hamiltonian symmetry group if $\{f, H\}=\omega\left(\underline{X}, X_{H}\right)=0$. The functions $f$ and $H$ are then said to Poisson commute. In this case, $f$ is a constant of the motion of $H$. To see this, simply compute

$$
0=\{f, H\}=\omega\left(\underline{X}, X_{H}\right)=i_{\underline{X}} \omega\left(X_{H}\right)=d f\left(X_{H}\right)=X_{H}(f) .
$$

This says that $f$ is constant along the integral curves of $X_{H}$. Similarly, $\underline{X}(H)=0$ so that $H$ is constant on orbits of the action. Let $M_{c}=f^{-1}(c)$ for $c \in \mathbb{R}$ a regular value and note that the $S^{1}$-action restricts to $M_{c}$. This follows since $f$ is constant on orbits of the action as well by

$$
\underline{X}(f)=d f(\underline{X})=i_{\underline{X}} \omega(\underline{X})=\omega(\underline{X}, \underline{X})=0 .
$$

Also, since $f$ is constant on the integral curves of $X_{H}$, we see that the portion of the $H$-flow starting in $M_{c}$ always remains in $M_{c}$. Thus, the Hamiltonian system restricts to $M_{c}$ with $\operatorname{dim} M_{c}=\operatorname{dim} M-1$. A reduced Hamiltonian system is then given on $M_{c}$ by $\tilde{H}: \tilde{M}=M_{c} / S^{1} \rightarrow \mathbb{R}$ and $\operatorname{dim} \tilde{M}=\operatorname{dim} M-2$. This process is called the MarsdenWeinstein Reduction. This reduction may be extended to other compact Lie groups. In fact, if $T^{n}$ acts as a Hamiltonian symmetry group on $\left(M^{2 n}, \omega\right)$, then, in principle, the Hamiltonian system may be solved by quadratures (i.e. successive integrations).

Another ingredient of symplectic geometry which we will need is the existence of an almost complex structure on a symplectic manifold. Recall that an almost complex structure on a manifold $M$ is an automorphism of the tangent bundle $J: T M \rightarrow T M$ such that $J^{2}=-1_{T M}$. Because $J$ behaves as the complex number $i$, it may be used to give the tangent vector spaces complex structures at each point of $M$. Of the spheres, only $S^{2}=\mathbb{C} P^{1}$ and $S^{6}$ have almost complex structures. Complex manifolds certainly 
have almost complex structures, but the existence of an almost complex structure does not necessarily mean that the underlying manifold is a complex manifold. An almost complex structure $J$ on a symplectic manifold $\left(M^{2 n}, \omega\right)$ is said to be compatible with $\omega$ if $\omega(J X, J Y)=\omega(X, Y)$ and $\omega(J X, X)>0$ for all vector fields $X$ and $Y$. The second condition allows us to define a Riemannian metric by $g(X, Y)=\omega(J X, Y)$.

Proposition 1.4. A symplectic manifold has a compatible almost complex structure.

Sketch of proof. Given a Riemannian metric $\langle-,-\rangle$, the nondegeneracy of $\omega$ allows us to define an isomorphism $A$ (on each tangent space) by $\omega(X, Y)=\langle X, A Y\rangle$. Then it is easy to see that $A$ is a normal and skew operator, so its polar decomposition $A=S J$ has $S J=J S$ with $S$ positive definite symmetric and $J$ an isometry. Furthermore, these properties and the skewness of $A$ give

$$
J^{t}=A^{t}\left(S^{-1}\right)^{t}=-A S^{-1}=-S J S^{-1}=-J .
$$

But $J$ is an isometry, so $J^{t}=J^{-1}$. Hence, $-J=J^{-1}$ and, therefore, $J^{2}=J\left(-J^{-1}\right)=-1$. Thus $J$ is an almost complex structure. Also, the definition of $A$ and the symmetricness of $S$ give

$$
\begin{aligned}
\omega(J X, J Y) & =\langle J X, A J Y\rangle=\left\langle J X, S J^{2} Y\right\rangle=\langle J X,-S Y\rangle=\langle S J X,-Y\rangle=\langle A X,-Y\rangle \\
& =-\langle Y, A X\rangle=-\omega(Y, X)=\omega(X, Y) .
\end{aligned}
$$

Thus, $J$ is an 'isometry' of $\omega$ also. Finally, define a new metric by

$$
\langle\langle X, Y\rangle\rangle=\langle S X, Y\rangle \text {. }
$$

This definition then makes sense because $S$ is positive definite symmetric in the old metric. Moreover, since $J$ is an isometry of the old metric and $\omega(X, Y)=\langle X, A Y\rangle$, we have

$$
\omega(J X, Y)=\langle J X, A Y\rangle=\langle J X, S J Y\rangle=\langle J X, J S Y\rangle=\langle X, S Y\rangle=\langle S X, Y\rangle=\langle\langle X, Y\rangle\rangle .
$$

In fact, a symplectic form determines a homotopy class of almost complex structures on $(M, \omega)$ and compatible structures can be found within this class. Therefore, when we refer to the Chern classes of a symplectic manifold, we are referring to those unchanging classes associated to any of the almost complex structures in the homotopy class. In particular (also see $\S 5$ ),

THEOREM 1.5 [McS]. If $S^{1}$ acts symplectically on a symplectic manifold $(M, \omega)$, then there exists an $S^{1}$-invariant almost complex structure in the homotopy class of structures determined by $\omega$. Hence, this invariant structure has the same Chern classes as any structure determined up to homotopy by $\omega$.

2. Some homotopy theory. As we shall see below, much of what can be done concerning circle actions on symplectic manifolds depends only on the homotopical structure of the manifold and the cohomology class (which we also denote simply by) $\omega \in H^{2}(M)$ given by the symplectic form. (When coefficients are not explicitly mentioned, we are taking real cohomology.) This leads us to

Definition 2.1. A manifold $M^{2 n}$ is cohomologically-symplectic (or c-symplectic) if there is a cohomology class $\omega \in H^{2}(M)$ such that $\omega^{n} \neq 0$. 
It is not the case that c-symplectic and symplectic are the same. For instance, if $M_{1}$ and $M_{2}$ are symplectic, then $M_{1} \# M_{2}$ is c-symplectic (since, in particular, the symplectic cohomology classes for each of $M_{1}$ and $M_{2}$ multiply up to top classes for $M_{1} \# M_{2}$ ). However, it is a general fact due to M. Audin [Au2] that, for $M_{1}$ and $M_{2}$ four-dimensional almost complex manifolds, the connected sum $M_{1} \# M_{2}$ is never almost complex - and, hence, cannot be symplectic. This distinction between symplectic and c-symplectic then gives somes meaning to our homotopical development of aspects of the theory of symplectic actions.

As we have said above, the obstruction to a circle action being Hamiltonian is the cohomology class represented by $i_{\underline{X}} \omega$ in $H^{1}(M)$. Let us attempt to view this obstruction from the point of view of algebraic topology.

An $S^{1}$-action and its orbit map may be generalized to produce certain basic elements in the fundamental group of the function space $\left(M^{M}, 1_{M}\right)$ in the following manner. By the exponential law, a group action $A: S^{1} \times M \rightarrow M$ corresponds to a map $\hat{\alpha}: S^{1} \rightarrow$ $\left(M^{M}, 1_{M}\right)$ with $\hat{\alpha}(s)(x)=A(s, x)$. Here, $\left(M^{M}, 1_{M}\right)$ denotes the path component of $M^{M}$ containing the identity map. The evaluation, $\operatorname{ev}(f)=f(p)$ of a function at a basepoint $m \in M$ gives ev $\circ \hat{\alpha}=\alpha \in \pi_{1}(M)$ and we then write $\operatorname{ev}_{\#}(\hat{\alpha})=\alpha$ where $e v_{\#}: \pi_{1}\left(M^{M}, 1_{M}\right) \rightarrow \pi_{1}(M)$. Furthermore, $\alpha$ may be identified with the homotopy class of the orbit map $a_{m}=A(-, m): S^{1} \rightarrow M$.

Now, because $A: S^{1} \times M \rightarrow M$ is an action, the adjoint map $\hat{\alpha}: S^{1} \rightarrow M^{M}$ is a monoid map which induces a map of classifying spaces $B S^{1} \rightarrow \operatorname{Baut}(M)$, where $\operatorname{Baut}(M)$ is the classifying space for fibrations with fibre $M$. It is known that

$$
\pi_{i+1}(\operatorname{Baut}(M)) \cong \pi_{i}\left(M^{M}, 1_{M}\right)
$$

for $i \geq 1$, so, at the $\pi_{2}$-level, $\hat{\alpha}$ corresponds to a de-looping $S^{2} \rightarrow B S^{1} \rightarrow \operatorname{Baut}(M)$. This map, in turn, provides a fibration

$$
M \stackrel{i}{\rightarrow} E \rightarrow S^{2}
$$

with $\alpha=\partial_{\#}(1) \in \operatorname{Im}\left(\partial_{\#}: \pi_{2} S^{2} \rightarrow \pi_{1} M\right)$. Such a fibration has a Wang sequence associated to it,

$$
\cdots \rightarrow H^{q}(E) \stackrel{i^{*}}{\rightarrow} H^{q}(M) \stackrel{\lambda_{\hat{\alpha}}}{\rightarrow} H^{q-1}(M) \rightarrow H^{q+1}(E) \rightarrow \cdots .
$$

The map $\lambda_{\hat{\alpha}}$ is a derivation on $H^{*}(M)$. That is, it satisfies

$$
\lambda_{\hat{\alpha}}(u v)=\lambda_{\hat{\alpha}}(u) v+(-1)^{|u|} u \lambda_{\hat{\alpha}}(v),
$$

and is called the Wang derivation. The factorization $S^{2} \rightarrow B S^{1} \rightarrow \operatorname{Baut}(M)$ then provides a homotopy-commutative diagram

$$
\begin{array}{ccccccc}
S^{1} & \rightarrow & M & \stackrel{j}{\rightarrow} & M S^{1} & \rightarrow & B S^{1} \\
\uparrow & & \| & & \uparrow \phi & & \uparrow \\
\Omega S^{2} & \rightarrow & M & \stackrel{i}{\rightarrow} & E & \rightarrow & S^{2}
\end{array}
$$

where $M S^{1}$ is the total space of the Borel fibration associated to the group action. We shall remind the reader of this fibration in a moment. Before we do, however, we point out that the Wang derivation may be related to the action in a very simple way (e.g. see [Sp]). 
Proposition 2.2. For any $u \in H^{q}(M)$,

$$
A^{*}(u)=1 \times u+\bar{\sigma} \times \lambda_{\hat{\alpha}}(u),
$$

where $\bar{\sigma} \in H^{1}\left(S^{1}\right)$ is a generator and $\times$ is the external product.

If $u \in H^{1}(M)$, then $A^{*}(u)=1 \times u+\alpha^{*}(u) \times 1$, where $\alpha=\mathrm{ev} \circ \hat{\alpha}$

Also, we should point out that (see $[\mathrm{G} 1,10.3])$, for $u \in H^{q}(M), \lambda_{\hat{\alpha}}(u)=h(\hat{\alpha}) \backslash \hat{E}^{*}(u)$, where \denotes slant product, $h: \pi_{1}\left(M^{M}, 1_{M}\right) \stackrel{\cong}{\rightarrow} H_{1}\left(M^{M} ; \mathbb{Z}\right)$ is the Hurewicz map and $\hat{E}: M^{M} \times M \rightarrow M$ is the evaluation map $\hat{E}(f, x)=f(x)$. Symplectic geometers actually were aware years ago of such a slant product description for the important symplectic invariant known as the Calabi invariant [Mc1].

For our purposes, the following simple observation is crucial.

Proposition 2.3. $\lambda_{\hat{\alpha}}(\omega)=0$ if and only if there exists $\bar{\omega} \in H^{2}(E)$ with $i^{*} \bar{\omega}=\omega$.

P r o of. This follows immediately from the exactness of the Wang sequence.

The $\lambda_{\hat{\alpha}}$-invariant is connected to symplectic geometry by the following fundamental result.

Theorem 2.4 [LO2]. If $\hat{\alpha} \in \pi_{1}\left(M^{M}, 1_{M}\right)$ comes from a symplectic $S^{1}$-action $A$ on a symplectic manifold $(M, \omega)$, then

$$
\lambda_{\hat{\alpha}}(\omega)=\left[i_{\underline{X}} \omega\right] .
$$

The theorem is proved by showing that

$$
A^{*} \omega=p_{2}^{*} \omega+p_{1}^{*} \nu \wedge p_{2}^{*} i_{\underline{X}} \omega
$$

where $\underline{X}$ is the fundamental vector field associated to the symplectic action $A$ and $\nu$ is a volume form on $S^{1}$. Theorem 2.4 is the first key to understanding Hamiltonian actions from a homotopical point of view. Theorem 2.4 also leads to

Definition 2.5. An $S^{1}$-action $A: S^{1} \times M \rightarrow M$ on a c-symplectic manifold $(M, \omega)$ is $c$-Hamiltonian if $\lambda_{\hat{\alpha}}(\omega)=0$ where $\hat{\alpha} \in \pi_{1}\left(M^{M}, 1_{M}\right)$ corresponds to $A$.

The second key to the homotopical approach is to understand the relation between $\lambda_{\hat{\alpha}}$ and the Borel fibration

$$
S^{1} \stackrel{a}{\rightarrow} M \stackrel{j}{\rightarrow} M S^{1} \rightarrow B S^{1} .
$$

Recall that, if a compact Lie group $G$, say, acts on a space $X$, then various symmetry properties of $X$ may be understood by studying the orbit space $X / G$ when $G$ acts freely. If the action is not free, then $X / G$ may have a quite intricate structure which is not easily described by homotopy theory. In the 1950's, Borel invented a substitute for the orbit space which is amenable to homotopical study, the so-called Borel fibration. To every $G$ there is associated a universal principal $G$-bundle

$$
G \rightarrow E G \rightarrow B G,
$$

where $E G$ is contactible with free $G$-action (so $B G=E G / G$ ). This bundle classifies principal $G$-bundles over a space $X$ in terms of the homotopy classes of maps $X \rightarrow B G$. 
Given an action $G \times X \rightarrow X,(g, x) \mapsto g x$, we can form the orbit space

$$
X G=\frac{E G \times X}{G}
$$

where $G$ acts diagonally $E G$ and $X$. Because $G$ acts freely on $E G$, it acts freely on $E G \times X$, so this orbit space is reasonable. There are two maps from $X G$ which tell us something about it. First, since elements of $X G$ are equivalence classes $[e, x]$, we can 'project' onto the equivalence class $[x] \in X / G$. It is not hard to show that this map $X G \rightarrow X / G$ is a homotopy equivalence when the action is free. In fact, a Leray spectral sequence argument shows that, if at each point $x$ the isotropy group of the action defined by $G_{x}=\{g \in G \mid g x=x\}$ is finite, then $X G \rightarrow X / G$ is a rational homotopy equivalence. Therefore, the Borel space $X G$ at least reduces homotopically to the right object under a free or almost free (i.e. finite isotropy) action. Secondly, we can project $[e, x]$ to the equivalence class $[e] \in B G$ to produce a fibration

$$
X \rightarrow X G \rightarrow B G
$$

called the Borel fibration. Good general references for the cohomology theory and (rational) homotopy theory of compact group actions are $[\mathrm{Br}]$ and $[\mathrm{AP}]$. Now, how does the Borel fibration for a symplectic $S^{1}$ action relate to the homotopy theory we have presented so far?

Consider an $S^{1}$ action on $M$ with $\hat{\alpha} \in \pi_{1}\left(M^{M}, 1_{M}\right)$ represented by the adjoint map of the action $\hat{\alpha}: S^{1} \rightarrow M^{M}$. The action is homotopically encoded in the Borel fibration (and its Barratt-Puppe extension with $\Omega B S^{1} \simeq S^{1}$ )

$$
S^{1} \stackrel{a}{\rightarrow} M \stackrel{j}{\rightarrow} M S^{1} \rightarrow B S^{1}
$$

Each fibration has an associated long exact sequence in homotopy and the diagram induces a commutative ladder

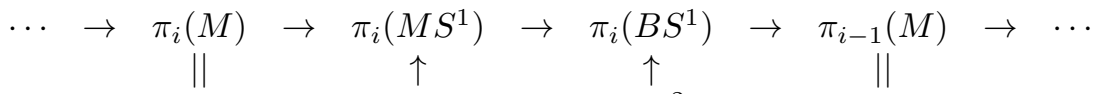

$$
\begin{aligned}
& \cdots \rightarrow \pi_{i}(M) \rightarrow \pi_{i}(E) \rightarrow \pi_{i}\left(S^{2}\right) \rightarrow \pi_{i-1}(M) \rightarrow \cdots .
\end{aligned}
$$

A simple diagram chase shows that, for $i=0,1$ or $2, \pi_{i} E \cong \pi_{i} M S^{1}$ and also $\pi_{3} E \rightarrow$ $\pi_{3} M S^{1}$ is surjective. Applying the Hurewicz theorem and duality, we obtain the following result.

Lemma 2.6. Let $A: S^{1} \times M \rightarrow M$ be an action. With the above notation, $\phi^{*}$ : $H^{r}\left(M S^{1}\right) \rightarrow H^{r}(E)$ is an isomorphism for $r \leq 2$.

For actions on a c-symplectic manifold, this has the following implication.

TheOREM 2.7. Let $A: S^{1} \times M \rightarrow M$ be an action on a c-symplectic manifold $\left(M^{2 n}, \omega\right)$. The action is c-Hamiltonian (i.e. $\left.\lambda_{\hat{\alpha}}(\omega)=0\right)$, if and only if there exists $\tilde{\omega} \in H^{2}\left(M S^{1}\right)$ with $j^{*} \tilde{\omega}=\omega$.

Proof. We have seen in Proposition 2.3 that $\lambda_{\hat{\alpha}}(\omega)=0$ if and only if there exists $\bar{\omega} \in H^{2}(E)$ with $i^{*} \bar{\omega}=\omega$. The isomorphism on $H^{2}$ obtained from Lemma 2.6 and the homotopy commutativity of the diagram (i.e. $\phi i \simeq j$ ) now imply the result. 
Now, in order to make explicit the differences in approach, let us consider a basic result about actions on symplectic manifolds from both the geometrical and homotopical viewpoints.

Proposition 2.8. A Hamiltonian or c-Hamiltonian action has fixed points.

Pro of 1. Assume the action is Hamiltonian and let $d f=i_{X} \omega$. Fixed points correspond to zeros of $\underline{X}$ and these correspond to critical points of $f$. The manifold $M$ is (as always) assumed to be compact, so $f$ attains its maximum and minimum. That is, critical points exist, so fixed points do also.

Pro of 2. Since the action is c-Hamiltonian, then $\lambda_{\hat{\alpha}}(\omega)=0$. By Theorem 2.7, there exists $\tilde{\omega} \in H^{2}\left(M S^{1}\right)$ with $j^{*} \tilde{\omega}=\omega$. Then we have $j^{*} \tilde{\omega}^{n}=\omega^{n} \neq 0$ so that $\tilde{\omega}^{n} \neq 0$ as well. Now suppose there is no fixed point so that the action is almost free (i.e. finite isotropy). Then $M S^{1} \rightarrow M / S^{1}$ is a rational equivalence so that, in particular, the image of $\tilde{\omega}^{n}$ must be nonzero in $H^{2 n}\left(M / S^{1}\right)$. But this is absurd since $\operatorname{dim} M / S^{1}=2 n-1$. Hence, fixed points exist.

REMARK 2.9. (1) The fact that $H^{i}\left(M^{2 n} / S^{1} ; \mathbb{Q}\right)=0$ for $i>2 n-1$ is not so easy to dig out in the literature, but a brief discussion of it, based on results in [Br], may be found in [Yau].

(2) Proposition 2.8 has many generalizations. Frankel [Fr] proved that if $M$ is Kähler, then an action is Hamiltonian if and only if there are fixed points. This has been generalized to the case of Lefschetz type by Ono [On1] and to the case of c-symplectic manifolds of Lefschetz type in [LO2]. For the notion of Lefschetz type, see $\S 3$ below.

The result above is just a simple application of topological notions to the geometry of Hamiltonian actions. More interesting applications may be found in [LO2]. For example, we mention the following result which is a mixture of the ideas presented above and work of Gottlieb [G1].

THEOREM 2.10. Suppose the first Chern class of a symplectic manifold $(M, \omega)$ is a positive multiple of $\omega$. That is, $c_{1}(M)=r \cdot \omega$ for $r>0$. Then any symplectic $S^{1}$ action on $M$ is Hamiltonian.

Sketch of proof. Without loss of generality, we take an $S^{1}$-invariant almost complex structure and associated Chern classes (which are the same as those for any other structure in the homotopy class determined by $\omega$ ). Because the almost structure is $S^{1}$-invariant, the map $\hat{\alpha}: S^{1} \rightarrow M^{M}$ associated to the action $A$ lifts to a map $S^{1} \rightarrow \operatorname{Bundle} \operatorname{Maps}(T M)$, where $T M$ is the tangent bundle of $M$. Gottlieb shows that this lift then factors through an essentially contactible space and that $k_{*} \circ \hat{\alpha}$ is nullhomotopic (i.e. homotopic to the constant map at $k$ denoted $\left.*_{k}\right)$. Here, $k: M \rightarrow B U(n)$ classifies $T M$ and $k_{*}:\left(M^{M}, 1_{M}\right) \rightarrow$ $\left(B U(n)^{M}, k\right)$ is the induced map given by $k_{*}(f)=k \circ f$. It is easy to see that the following diagram commutes.

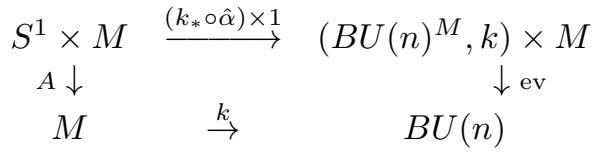


Here, ev is the evaluation map defined by $\operatorname{ev}(f, m)=f(m)$. This diagram then gives

$$
k \circ A \simeq \operatorname{ev}\left(\left(k_{*} \circ \hat{\alpha}\right) \times 1\right) \simeq \operatorname{ev}\left(*_{k} \times 1\right) \simeq k \circ p_{2}
$$

where $p_{2}: S^{1} \times M \rightarrow M$ is projection onto the second factor. Then, for any class $c \in H^{*}(B U(n))$,

$$
A^{*} k^{*}(c)=p_{2}^{*} k^{*}(c)=1 \times k^{*}(c) .
$$

By our description of $\lambda_{\hat{\alpha}}$, this means that $\lambda_{\hat{\alpha}}\left(k^{*}(c)\right)=0$. But the Chern classes of $M$ come from universal Chern classes in $H^{*}(B U(n))$, so $\lambda_{\hat{\alpha}}$ vanishes on all Chern classes of $M$. If $c_{1}(M)=r \cdot \omega$, then $\lambda_{\hat{\alpha}}(\omega)=0$ as well and $A$ is Hamiltonian.

REMARK 2.11. In [On3], K. Ono shows that there are no nontrivial symplectic actions of compact connected Lie groups on $M$ when $c_{1}(M)=r \omega$ for $r<0$. Thus, the condition $r>0$ in the theorem is to ensure nontriviality.

Theorem 2.10 may also be used to obtain conditions when certain results in symplectic geometry can be made $S^{1}$-equivariant. It is a standard fact in symplectic geometry ([Ti]) that if $\left(M^{2 n}, \omega\right)$ is a symplectic manifold with integral form $\omega$ (i.e. $[\omega] \in H^{2}(M ; \mathbb{Z}) \subset$ $\left.H^{2}(M)\right)$, then there is an embedding $\phi: M \hookrightarrow \mathbb{C} P^{N}$ with $N=2 n+1$ and $\phi^{*}\left(\omega_{0}\right)=\omega$, where $\omega_{0}$ is the standard Kähler form on $\mathbb{C} P^{2 n+1}$.

Let $\phi^{*} T \mathbb{C} P^{N}$ be the pullback of the tangent bundle of $\mathbb{C} P^{N}$ by the embedding $\phi$. Then, denoting the normal bundle of the embedding by $\nu$, we have $\phi^{*} T \mathbb{C} P^{N}=T M \oplus \nu$. Also, $\nu$ is a complex bundle just as $T M$ and $T \mathbb{C} P^{N}$ are. The reason for this is that the normal bundle $\nu \subset T \mathbb{C} P^{N}$ is the $\omega$-orthogonal complement to $T M \subset T \mathbb{C} P^{N}$ and $\omega$ thus restricts to give a skew-symmetric bilinear (i.e. symplectic) form on $\nu$. Since $U(n+1) \simeq S p(2 n+2, \mathbb{R})$, this gives the required reduction of the structure group to $U(n+1)$.

THEOREM 2.12. Suppose $(M, \omega)$ is a symplectic manifold with integral form $\omega$. If $c_{1}(\nu)=0$, then, for any symplectic $S^{1}$-action on $(M, \omega)$, there is an $S^{1}$-equivariant symplectic embedding $\theta: M \hookrightarrow \mathbb{C} P^{K}$ (i.e $\left.\theta^{*}\left(\omega_{0}\right)=\omega\right)$ for large enough $K$.

Proof. Let $L$ denote the complex line bundle given by $c_{1}\left(L^{*}\right)=[\omega]$. Ono [On1] shows that such an equivariant embedding exists if the $S^{1}$-action may be lifted to $L$. But, using the Hattori-Yoshida lifting theorem [HY], Gottlieb [G3] showed that such a lifting exists exactly when $\lambda_{\hat{\alpha}}(\omega)=0$. (As usual, we write $\omega$ for both the symplectic form and its cohomology class, but the context should be clear.) Therefore, we shall prove that this holds under the assumption $c_{1}(\nu)=0$. First, note that

$$
\begin{aligned}
c\left(\phi^{*} T \mathbb{C} P^{N}\right) & =c(T M) \cdot c(\nu) \\
& =\left(1+c_{1}(M)+c_{2}(M)+\ldots\right) \cdot\left(1+c_{1}(\nu)+c_{2}(\nu)+\ldots\right) .
\end{aligned}
$$

Hence, $c_{1}\left(\phi^{*} T \mathbb{C} P^{N}\right)=c_{1}(M)+c_{1}(\nu)=c_{1}(M)$ by hypothesis. Of course, $c\left(\mathbb{C} P^{N}\right)=$ $\left(1+\omega_{0}\right)^{N+1}$, so $c_{1}\left(\mathbb{C} P^{N}\right)=(N+1) \omega_{0}$. Also,

$$
c_{1}\left(\phi^{*} T \mathbb{C} P^{N}\right)=\phi^{*}\left(c_{1}\left(\mathbb{C} P^{N}\right)\right)=\phi^{*}\left((N+1) \omega_{0}\right)=(N+1) \omega .
$$

Thus, $c_{1}(M)=(N+1) \omega$ and, by Theorem 2.10 , any symplectic $S^{1}$-action has $\lambda_{\hat{\alpha}}(\omega)$ $=0$. 
We have given only the barest hint of the homotopical approach to Hamiltonian actions above. In the next section, we will see how the c-Hamiltonian approach provides a solution to one case of a conjecture mixing symplectic geometry, group actions and rational homotopy theory.

3. Nilmanifolds of Lefschetz type. Thurston [Th] (and, independently, Kodaira) gave the first example of a symplectic non-Kähler manifold by defining a certain 4dimensional nilmanifold $K T$ which he showed was symplectic with first Betti number three. As in Example 1.1 (4), the Hard Lefschetz property for Kähler manifolds requires odd Betti numbers to be even, so the Kodaira-Thurston manifold could not be Kähler. After this example, the problem became to find examples of simply connected symplectic non-Kähler manifolds and this proved much harder. Finally, in 1984, McDuff [Mc2] found such examples via a type of blow-up construction. (More, recently, R. Gompf has used symplectic surgery to create a host of new examples.) For more on this question of symplectic versus Kähler, see [TO]. To see how this question relates to the $\lambda_{\hat{\alpha}}$-invariant, we need to review several things.

First, because there are symplectic manifolds which are not Kähler, it is often the case that we wish to compare homotopical properties of symplectic and Kähler manifolds. In this way, we obtain an algebraic measure of the disparity in the two classes of manifolds. With this in mind, we make the

Definition 3.1. A c-symplectic manifold has Lefschetz type if multiplication by $\omega^{n-1}$ is an isomorphism from $H^{1}(M)$ to $H^{2 n-1}(M)$.

EXAMPLE 3.2. A general way to obtain non-Kählerian, symplectic manifolds of Lefschetz type is as follows. Let $\left(X^{2 m}, \omega_{X}\right)$ be a Kähler manifold and let $\left(Y^{2 n}, \omega_{Y}\right)$ be a simply-connected symplectic manifold. Their product $X \times Y$ is a $(2 m+2 n)$-dimensional symplectic manifold with symplectic class $\omega_{X}+\omega_{Y}$. This manifold is clearly of Lefschetz type. Suppose in addition that the symplectic manifold $Y$ has some odd Betti number that is odd. Then so too does the product $X \times Y$. For if $b_{2 i+1}(Y)$ is the first odd Betti number which is odd, then

$$
\begin{gathered}
b_{2 i+1}(X \times Y)=b_{2 i+1}(X)+b_{2 i}(X) b_{1}(Y)+b_{2 i-1}(X) b_{2}(Y)+ \\
\ldots+b_{1}(X) b_{2 i}(Y)+b_{2 i+1}(Y) .
\end{gathered}
$$

Since $X$ is Kähler, each $b_{2 j+1}(X)$ is even and, by assumption, each $b_{2 j+1}(Y)$ for $j<i$ is even. Hence each term in the righthand sum except the last, which is odd, is even and so $b_{2 i+1}(X \times Y)$ is odd. It follows that $X \times Y$ cannot admit a Kähler structure. Furthermore, if $X$ is a Kähler manifold and if $Y$ is a symplectic manifold of Lefschetz type, then $X \times Y$ is again of Lefschetz type. As previously, if $Y$ has an odd Betti number that is odd, so does the product, which therefore cannot admit a Kähler structure. Starting with McDuff's examples [Mc2] of simply-connected, symplectic manifolds that have an odd Betti number which is odd, we thus obtain many examples of symplectic manifolds of Lefschetz type that cannot admit a Kähler structure.

Second, recall that a nilmanifold $\mathcal{N} / \pi$ of dimension $2 k$ is the quotient of a nilpotent Lie group $\mathcal{N}^{2 k}$ by a discrete subgroup $\pi$ so that $\mathcal{N} / \pi$ is compact. An $n$-dimensional 
nilpotent Lie group is diffeomorphic to $\mathbb{R}^{n}$, so

$$
\mathcal{N} / \pi \simeq K(\pi, 1) .
$$

Then, since $\mathcal{N} / \pi$ is compact, $\pi$ is finitely generated torsionfree nilpotent. Indeed, by Malcev's work, every such group arises as the fundamental group of a nilmanifold. Now, in the 1950's Nomizu [No] proved that $\pi$-invariant forms on $\mathcal{N}$ contain the left invariant forms on $\mathcal{N}$ so as to induce an isomorphism on cohomology. In other words,

$$
H^{*}\left(\Omega_{\mathcal{N}} \mathcal{N}\right) \stackrel{\cong}{\longrightarrow} H^{*}\left(\Omega_{\pi} \mathcal{N}\right) \cong H^{*}(\mathcal{N} / \pi) .
$$

But the left invariant forms $\Omega_{\mathcal{N}} \mathcal{N}$ may be identified with the cochain complex $\Lambda^{*} \mathfrak{n}$ of the Lie algebra $\mathfrak{n}$ of $\mathcal{N}$. Now, $\Lambda^{*} \mathfrak{n}$ is an exterior algebra, so, by the identifications above, if $\omega \in$ $H^{*}(\mathcal{N} / \pi)$ with $\omega^{k} \neq 0$, then there is a degree two exterior form $\beta$ in $\Lambda^{*} \mathfrak{n}$ which has $\beta^{k} \neq 0$ in $\Lambda^{*} \mathfrak{n}$. But in an exterior algebra, this must mean that the 2 -form is nondegenerate. This is essentially the same argument as that which gives the equivalence of nondegeneracy of symplectic forms with the property of wedging to a volume form. Thus, the corresponding 2 -form on $\mathcal{N} / \pi$ is nondegenerate as well and $\mathcal{N} / \pi$ is symplectic. Therefore, c-symplectic and symplectic mean the same thing for nilmanifolds.

On the algebraic side, there is a refinement of the upper central series of $\pi$,

$$
\pi \supseteq \pi_{2} \supseteq \pi_{3} \supseteq \cdots \supseteq \pi_{n} \supseteq 1,
$$

with each $\pi_{i} / \pi_{i+1} \cong \mathbb{Z}$. The length of this series is invariant and is called the rank of $\pi$. So, for $\pi$ above, $\operatorname{rank}(\pi)=n$. This description implies that any $u \in \pi$ has a decomposition $u=u_{1}^{x_{1}} \cdots u_{n}^{x_{n}}$, where $\left\langle u_{n}\right\rangle=\pi_{n}, \cdots\left\langle u_{i}\right\rangle=\pi_{i} / \pi_{i+1}$. The set $\left\{u_{1}, \cdots u_{n}\right\}$ is called a Malcev basis for $\pi$. Using this basis, the multiplication in $\pi$ takes the form

$$
u_{1}^{x_{1}} \cdots u_{n}^{x_{n}} u_{1}^{y_{1}} \cdots u_{n}^{y_{n}}=u_{1}^{\rho_{1}(x, y)} \cdots u_{n}^{\rho_{n}(x, y)}
$$

where

$$
\rho_{i}(x, y)=x_{i}+y_{i}+\tau_{i}\left(x_{1}, \ldots x_{i-1}, y_{1}, \ldots y_{i-1}\right) .
$$

For instance, we may consider the Heisenberg subgroup $\mathcal{N}=U_{n}(\mathbb{R})$, the group of upper triangular matrices with 1 's on the diagonal, and take $\pi=U_{n}(\mathbb{Z})$, such matrices with integral entries. A Malcev basis is given by $\left\{u_{i j} \mid 1 \leq i<j \leq n\right\}$ where $u_{i j}=I+e_{i j}$ and

$$
\rho_{i j}(x, y)=x_{i j}+y_{i j}+\sum_{i<k<j} x_{i k} y_{k j} .
$$

Now, consider the central extension $\pi_{n} \rightarrow \pi \rightarrow \bar{\pi}$. The cocycle for the extension is $\tau_{n}: \bar{\pi} \times \bar{\pi} \rightarrow \mathbb{Z}$. Of course $\bar{\pi}$ is also finitely generated torsionfree with refined upper central series,

$$
\bar{\pi}=\frac{\pi}{\pi_{n}} \supseteq \frac{\pi_{2}}{\pi_{n}} \supseteq \cdots \supseteq \frac{\pi_{n-1}}{\pi_{n}} \supseteq \frac{\pi_{n}}{\pi_{n}}=1 .
$$

Hence, $\operatorname{rank}(\bar{\pi})=n-1$ and

$$
\bar{\rho}_{i}(x, y)=\rho_{i}((x, 0),(y, 0))=x_{i}+y_{i}+\tau_{i}\left(x_{1}, \ldots x_{i-1}, y_{1}, \ldots y_{i-1}\right)
$$

for $i<n$. Clearly, then, we may iterate this process and decompose $\pi$ as $n$ central extensions of the form

$$
\mathbb{Z} \rightarrow G \rightarrow \bar{G}
$$


with cocycles $\tau_{i} \in H^{2}(\bar{G} ; \mathbb{Z})$ (where the coefficients are untwisted since the extension is central).

This description allows a geometric formulation. The extension class $\tau_{n}$ is an element of $H^{2}(\bar{\pi} ; \mathbb{Z}) \cong H^{2}(K(\bar{\pi}, 1) ; \mathbb{Z}) \cong[K(\bar{\pi}, 1), K(\mathbb{Z}, 2)]$ where the last bijection follows from the usual identification of cohomology groups with sets of homotopy classes into $K(\mathbb{Z}, m)$ 's. Now, $K(\mathbb{Z}, 2)=\mathbb{C} P^{\infty}$, the classifying space for principal $S^{1}$-bundles, so $\tau_{n}$ induces a bundle over $K(\bar{\pi}, 1)$,

$$
\begin{aligned}
S^{1} \longrightarrow & K(\pi, 1) \\
& \downarrow \\
& K(\bar{\pi}, 1) \stackrel{\tau_{n}}{\longrightarrow} \mathbb{C} P^{\infty} .
\end{aligned}
$$

The total space of the bundle is clearly $K(\pi, 1)$ since the ensuing short exact sequence of fundamental groups in classified by $\tau_{n}$. Now, because we can iterate the algebraic decomposition of $\pi$, we obtain an iterated sequence of principal $S^{1}$-bundles classified by the $\tau_{i}$.

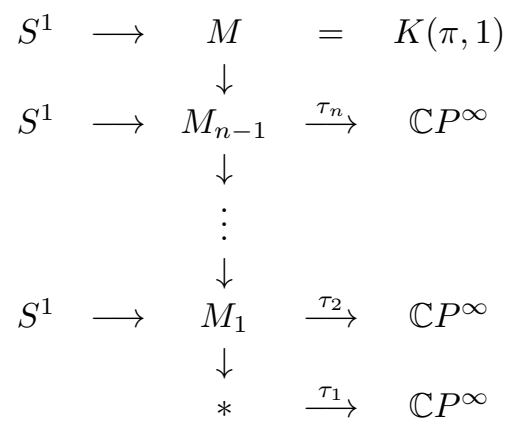

All along, we have only been interested in real (or de Rham) cohomology, so it should be no surprise that our approach to understanding symplectic nilmanifolds comes from rational or real homotopy theory. With this in mind, recall that, to any space $X$ with finite-type rational homology, is associated a minimal model $(\Lambda V, d)$, where $V$ is a positively graded vector space and $\Lambda V$ is a freely generated (commutative) differential graded algebra (DGA) which is polynomial on even degree generators, exterior on odd degree generators and which has a decomposable differential $d$. The minimal model of $X$ is constructed from the commutative differential graded algebra of rational polynomial forms $A^{*}(X)$, akin to de Rham forms on a smooth manifold. Indeed, if we are interested in real homotopy type, then we may take the minimal model of the de Rham forms as the model of $X$.

A nilpotent space is one whose fundamental group is nilpotent and whose fundamental group acts nilpotently on higher homotopy. For example, an $H$-space or a simply connected space is nilpotent. More important for us, however, is the fact that a nilmanifold is clearly a nilpotent space. The fundamental theorem of rational homotopy theory asserts that each nilpotent space $X$ has a minimal model which contains all the rational homotopy information about the space. In particular, for nilpotent $X$, there is a natural isomorphism $H^{*}(X ; \mathbb{Q}) \cong H^{*}((\Lambda V, d))$ and $\operatorname{Hom}\left(\pi_{*}(X), \mathbb{Q}\right)$ may be read off from $V$. For details, see [Su], [GM]. 
More precisely, the minimal model for a space is constructed in a stage by stage fashion which precisely mirrors the rational Postnikov tower of the space. In fact, the differential $d$ of the model corresponds to the $k$-invariant in the tower. In particular, the decomposition of the nilmanifold $M=K(\pi, 1)$ into a tower of principal $S^{1}$-bundles is, in fact, the Postnikov decomposition of $M$ with $k$-invariants the $\tau_{i}$. With this in mind, we have

Proposition 3.3. The minimal model of a nilmanifold has the form,

$$
\Lambda(M)=\left(\Lambda\left(x_{1}, \ldots x_{n}\right), d\right) \quad \text { with } \quad\left|x_{i}\right|=1,
$$

where $d x_{i}=\tau_{i}$, the extension cocycle representing the class $\tau_{i} \in H^{2}\left(M_{i-1} ; \mathbb{Z}\right)$. Note that $\Lambda(M)$ is an exterior algebra because all generators are in odd degree 1.

ExAmples 3.4. (1) The torus $T^{n}$. The minimal model is $\left(\Lambda\left(x_{1}, x_{2}, \ldots, x_{n}\right), d=0\right)$. If $n=2 k$, then $T^{n}$ is symplectic by Example 1.1(3) and a symplectic 'form' in the minimal model is $\omega=x_{1} x_{k+1}+\ldots+x_{k} x_{2 k}$.

(2) The Heisenberg manifold. From the description above of the Heisenberg subgroup $U_{3}(\mathbb{Z})$, we see that a minimal model for the associated nilmanifold is given by

$$
\Lambda\left(U_{3}(\mathbb{Z})\right)=(\Lambda(x, y, z), d x=0, d y=0, d z=x y) .
$$

(3) The torsionfree nilpotent group

$$
\pi=\left\langle x, y, z:[x, y]=z^{2},[x, z]=e=[y, z]\right\rangle,
$$

has, by a Malcev basis argument, $\tau=2 x y$. Hence, a minimal model for $K(\pi, 1)$ is given by

$$
\Lambda(\pi)=(\Lambda(x, y, z), d x=0, d y=0, d z=2 x y) .
$$

(4) The Kodaira-Thurston manifold KT. Rationally, this manifold is obtained by taking the product of the Heisenberg manifold $M=U_{3}(\mathbb{R}) / U_{3}(\mathbb{Z})$ and the circle $S^{1}$. The minimal model is then given by

$$
\left(\Lambda\left(x_{1}, x_{2}, x_{3}, x_{4}\right), d\right) \quad \text { with } \quad d x=0, d y=0, d z=x y, d u=0
$$

because of the general fact that the minimal model of a product is the tensor product of the minimal models of the factors. A symplectic 'form' is then given by $\omega=x u+y z$. Note that the degree 1 cohomology of $K T$ is generated by the classes of $x, y$ and $u$. Hence, the first Betti number is three and $K T$ cannot be Kähler.

(5) Take the minimal model $\left(\Lambda\left(x_{1}, x_{2}, x_{3}, x_{4}\right), d\right)$ with $d x=0, d y=0, d z=x y$ and $d u=x z$. Recall that the corresponding finitely generated torsionfree nilpotent group may be realized as a nilmanifold. Then a symplectic 'form' is given by $\omega=x u+y z$.

REMARK 3.5. The torus $T^{4}$ and Example 3.4 (4) and (5) are all the possible real homotopy types of 4-dimensional nilmanifolds. Thus, all 4-dimensional nilmanifolds admit symplectic structures (see below also).

There is one other important feature about minimal models of nilmanifolds which should be emphasized. Namely, there are precisely $n$ degree 1 generators for an $n$ dimensional nilmanifold. Because the minimal model is an exterior algebra (so that the square of each generator vanishes), there is only one element which can represent the top 
class in cohomology. That element is the product of all generators, $\nu=x_{1} \cdots x_{n}$. Now let's tie in the symplectic geometry of nilmanifolds with the minimal model.

If a nilmanifold $M^{2 k}$ is symplectic (which is the same as c-symplectic remember), then there must be a degree 2 element of the minimal model which multiplies up to a multiple of the top element $\nu=x_{1} \cdots x_{2 k}$. By changing the basis of degree 1 generators if necessary, we can write this degree 2 element as

$$
\omega=\sum x_{i} \cdot x_{j}
$$

Since $\omega^{k}=\nu$, the sum in the expression for $\omega$ must contain all the degree 1 generators. Also, recall that the symplectic 'form' $\omega$ must be closed. The following series of results focuses on the question of what Kähler properties symplectic nilmanifolds can have.

TheOREM 3.6 ([BG], [Has]). Let $M=N / \Gamma$ be a compact nilmanifold. If $M$ admits a Kähler structure, it is diffeomorphic to a torus.

Sketch of proof. We have seen that the minimal model of $M$ is an exterior algebra $\Lambda\left(x_{1}, \ldots, x_{n}\right)$ with top class represented by the cocycle $\nu=x_{1} \cdots x_{n}$. Now, except when $d x_{i}=0$ for all $i=1, \ldots, n$, no matter how we write the generators of the model, $\nu$ is always an element of the ideal $\operatorname{Ideal}(N)$ generated by the complement $N$ to the closed degree 1 generators. In rational homotopy theory, this is what is meant by saying that $\nu$ is a non-vanishing Massey product. The main result of [DGMS] says that all Massey products vanish (uniformly) for a Kähler manifold. Hence, $M$ cannot be Kähler unless $d=0$ and then $M$ has the rational homotopy type of a torus.

Now, since the fundamental group $\pi_{1}(M)$ of a nilmanifold is torsionfree and $\mathbb{Q}$ localization has torsion kernel, $\pi_{1}(M)$ must inject into $\oplus \mathbb{Q}$. Hence, since $\pi_{1}(M)$ is finitely generated and injects into an abelian torsionfree group, it must be the case that $\pi_{1}(M) \cong$ $\oplus \mathbb{Z}$ and $M$ has the homotopy type of a torus. But $M$ is a true nilmanifold, so its fundamental group classifies its diffeomorphism type ([Mos]). Therefore, $M$ is diffeomorphic to a torus.

In fact, however, the hypothesis of Theorem 3.6 is too strong. Recall from Definition 3.1 that a (cohomologically) symplectic manifold $M$ has Lefschetz type if

$$
[\omega]^{n-1} \cdot: H^{1}(M) \rightarrow H^{2 n-1}(M)
$$

is an isomorphism. Just as we did before, we will now give a geometric and an algebraic proof for the following extension of Theorem 3.6.

TheOrem 3.7 ([BG], [Mc3], [LO1], [LO2]). A symplectic nilmanifold $M$ of Lefschetz type is diffeomorphic to a torus.

Proof 1 [Mc3]. Without loss of generality, we can assume the symplectic form on $M=N / \pi$ comes from a left invariant form on the nilpotent Lie group $N$. Now, the center of $\pi$ is contained in the center of $N$, so each element of the center of $\pi$ gives rise to a free symplectic circle action on $M$. If $\pi$ is nilpotent but not abelian, then there exists [Wa] an element $\alpha$ in the intersection of the center of $\pi$ and the commutator subgroup $[\pi, \pi]$. But then we have a free (so without fixed points) circle action with $\iota_{*}\left(\nu_{S^{1}}\right)=\alpha=0$ since $H_{1}(M)=\pi /[\pi, \pi]$. This contradicts the fact due to Ono [On1] (and generalized in 
[LO2]) that, in the presence of the Lefschetz type condition, a symplectic circle action $S^{1} \times M \rightarrow M$ on a closed symplectic manifold $M$ has fixed points if and only if the orbit map (restricting the action to a fixed $\left.m_{0} \in M\right) \iota: S^{1} \rightarrow M$ is trivial on homology. Hence, the non-abelian hypothesis is incorrect and $M$ is a torus.

Pr o of 2 [LO1]. We have seen that the minimal model for $M$ is $\left(\Lambda\left(x_{1}, \ldots, x_{2 n}\right), d\right)$ with symplectic 'form' given by

$$
\omega=\sum_{i, j<2 n} a_{i j} x_{i} x_{j}+z x_{2 n}
$$

where we have taken out all terms involving the last generator $x_{2 n}$. Assume that $d \neq 0$. First note that, because of the stage-by-stage construction of the minimal model, the last generator $x_{2 n}$ cannot appear in any differential $d x_{i}, i=1, \ldots, 2 n$. Hence, since $d \omega=0$, the only way to have the term $d z \cdot x_{2 n}$ cancelled is for $z$ to be a cocycle, $d z=0$. Now define a derivation $\lambda$ of degree -1 by

$$
\lambda\left(x_{i}\right)=0 \quad \text { for } i<2 n, \quad \lambda\left(x_{2 n}\right)=1 .
$$

Extend $\lambda$ freely to $\left(\Lambda\left(x_{1}, \ldots, x_{2 n}\right), d\right)$. The effect of $\lambda$ on $\omega$ follows from the definition and the derivation property:

$$
\lambda(\omega)=z .
$$

The derivation $\lambda$ obeys the relation $\lambda d=-d \lambda$ because $d$ is decomposable, so a derivation of cohomology is induced with $\lambda([\omega])=[z] \neq 0$ since $z$ is a non-zero degree 1 cocycle.

Now, a basis for $H^{1}(M)$ consists precisely of the generators $x_{1}, \ldots, x_{s}$ with $d x_{i}=0$, $i=1, \ldots, s$. By the definition of $\lambda$ then, $\lambda\left(H^{1}(M)\right)=0$. Let $[\alpha]$ be any element of $H^{1}(M)$ and consider $[\alpha] \cup\left[\omega^{n}\right]$. Since this class is above the top degree, $[\alpha] \cup\left[\omega^{n}\right]=0$. Applying $\lambda$, we obtain

$$
0=\lambda\left([\alpha] \cup\left[\omega^{n}\right]\right)=\lambda([\alpha]) \cup\left[\omega^{n}\right]-[\alpha] \cup \lambda\left(\left[\omega^{n}\right]\right)=-[\alpha] \cup \lambda\left(\left[\omega^{n}\right]\right) .
$$

Now $[\alpha] \cup \lambda\left(\left[\omega^{n}\right]\right)=0$ for any $[\alpha] \in H^{1}(M)$, where $\lambda\left(\left[\omega^{n}\right]\right) \in H^{2 n-1}(M)$. By Poincaré duality (i.e. the nondegeneracy of the bilinear form), this can only be true if $\lambda\left(\left[\omega^{n}\right]\right)=0$. But then

$$
0=\lambda\left(\left[\omega^{n}\right]\right)=n \lambda([\omega]) \cup\left[\omega^{n-1}\right]
$$

and the hypothesis of Lefschetz type implies that this can happen only when $\lambda([\omega])=0$. This contradicts the equation $\lambda([\omega])=[z] \neq 0$ derived above. The contradiction lies in the assumption that $d \neq 0$, so we see that a Lefschetz type nilmanifold must have $d=0$ and, so, have the rational homotopy type of a torus.

Now, the same argument as in the final paragraph of the proof of Theorem 3.6 shows that $M$ is diffeomorphic to a torus.

REMARK 3.8 (Connections between proofs 1 and 2). The construction of the minimal model from the decomposition of the fundamental group of the nilmanifold shows that $x_{2 n}$ (as well as the dual derivation $\lambda$ ) corresponds to an element in the intersection of the center of $\pi$ and the commutator subgroup of $\pi$. This is McDuff's element. For any aspherical $M$, it is known that $\pi_{1}\left(M^{M}, 1_{M}\right) \cong \mathcal{Z} \pi$, the center of $\pi$. Hence, this central element corresponds to an $\hat{\alpha}$ and the derivation $\lambda$ corresponds to the associated $\lambda_{\hat{\alpha}}$. 
Proof 2 then shows that the $S^{1}$-action given by the central element is not Hamiltonian (since $\lambda_{\hat{\alpha}}(\omega)=z \neq 0$ ), but $\alpha_{*}=0$ (where $\alpha_{*}$ is the homomorphism on homology induced by the orbit map $\left.\alpha: S^{1} \rightarrow M\right)$. As mentioned in Proof 1 , the following equivalences hold when any $M$ (i.e. not necessarily aspherical) has Lefschetz type (see [LO2, §3]):

$$
\lambda_{\hat{\alpha}}(\omega)=0 \Leftrightarrow \lambda_{\hat{\alpha}}\left(\omega^{n}\right)=0 \Leftrightarrow \lambda_{\hat{\alpha}}\left(H^{1}(M)\right)=0 \Leftrightarrow h(\alpha)=0
$$

where $h(\alpha)$ is the Hurewicz homomorphism applied to the orbit map. So, the central element, being zero on homology, would imply that the action is Hamiltonian. But Hamiltonian actions, as we have seen, have fixed points, while the action is free. This Theorem 3.7 is truly a result where geometry is reflected clearly in algebraic structure. Because of this, a result such as Theorem 3.7 may be generalized. For such a generalization, see [LO2, Corollary 4.6].

4. One more comparison. As a final comparison of the geometric and algebraic approaches to the subject of circle actions on symplectic manifolds, we shall consider the following (see [On2] and [LO2])

Theorem 4.1 [On2]. If $\left.\omega\right|_{\pi_{2}(M)}=0$, then any symplectic circle action on $M$ has no fixed points (and, so, is not Hamiltonian).

Here we think of the symplectic cohomology class $\omega$ as a dual element of degree 2 homology, $\omega \in \operatorname{Hom}\left(H_{2}(M), \mathbb{R}\right)$. The Hurewicz map $h: \pi_{2}(M) \rightarrow H_{2}(M)$ has image in degree 2 homology and the notation $\left.\omega\right|_{\pi_{2}(M)}=0$ simply means that $\omega$ is zero on this image. We shall present two sketches of proofs of this result, one geometric and the other algebraic. Let $A: S^{1} \times M \rightarrow M$ be a symplectic circle action on a symplectic manifold $(M, \omega)$ with $\left.\omega\right|_{\pi_{2}(M)}=0$. (Without loss of generality we assume the action is effective.)

Proof 1 (sketch) [On2]. Choose a path $\gamma:[0,1] \rightarrow M$ with $\gamma(0), \gamma(1) \in \operatorname{Fix}(A)$ and $i_{X} \omega(\dot{\gamma})>0$ for each $t \in[0,1]$. (Note that the existence of such a $\gamma$ requires some proof.) Define a 2-cycle $C: S^{1} \times[0,1] \rightarrow M$ by $C(s, t)=A(s, \gamma(t))$. The homology class $[C]$ is in the image of the Hurewicz homomorphism and, by hypothesis, $\langle\omega,[C]\rangle=0$. But,

$$
\langle\omega,[C]\rangle=\int_{C} \omega=\int_{\gamma} i_{\underline{X}} \omega=\int_{0}^{1} i_{\underline{X}} \omega(\dot{\gamma}) d t>0 .
$$

This contradiction then shows that no fixed points can exist.

Pro of 2 [LO2]. We have the following facts:

(1) For $\pi_{1}(M)=\pi$, there is a classifying map $f: M \rightarrow K(\pi, 1)$ such that

$$
H_{1}(M ; \mathbb{Z}) \cong H_{1}(\pi ; \mathbb{Z}) \quad \text { and } \quad H_{2}(M ; \mathbb{Z}) / \operatorname{Image}(h) \cong H_{2}(\pi ; \mathbb{Z})
$$

where the homology of $\pi$ is that of $K(\pi, 1)$ and $h$ is the degree 2 Hurewicz homomorphism. This is a result due to Hopf and is easily proved.

(2) The condition $\left.\omega\right|_{\pi_{2}(M)}=0$ translates into the condition that there exists an element $\omega_{\pi} \in H^{2}(\pi)$ with $f^{*} \omega_{\pi}=\omega$. This follows from (1). (Here, as usual, cohomology with no explicit coefficients denotes real cohomology.)

(3) Let $\alpha: S^{1} \rightarrow M$ denote the orbit map of the action $A$ and let $\pi^{\prime}=\pi /\langle\alpha\rangle$. A special case of a theorem of Browder and Hsiang $[\mathrm{BH}]$ asserts that there is a commutative diagram 


$$
\begin{array}{cccc}
H^{*}(M) & \stackrel{f^{*}}{\leftarrow} & H^{*}(K(\pi, 1)) \\
q^{*} \uparrow & & \uparrow p^{*} \\
H^{*}\left(M / S^{1}\right) & \stackrel{g}{\leftarrow} & H^{*}\left(K\left(\pi^{\prime}, 1\right)\right) .
\end{array}
$$

where $q: M \rightarrow M / S^{1}$ is the orbit space quotient. (As noted in [BH], the homomorphism $g$ may not be induced by a map of spaces.)

To complete the proof using these facts, first note that, if there exist fixed points, then $\langle\alpha\rangle=0$, so $\pi^{\prime}=\pi$. Therefore, the diagram above becomes

$$
\begin{array}{ccc}
H^{*}(M) & \stackrel{f^{*}}{\leftarrow} & H^{*}(K(\pi, 1)) \\
q^{*} \uparrow & \swarrow g & \\
H^{*}\left(M / S^{1}\right) & &
\end{array}
$$

with $q^{*}\left(g\left(\omega_{\pi}\right)\right)=f^{*}\left(\omega_{\pi}\right)$. Now, $g\left(\omega_{\pi}^{n}\right)=0$ since $\operatorname{dim}\left(M / S^{1}\right)<2 n$, so $0=q^{*}\left(g\left(\omega_{\pi}^{n}\right)\right)=$ $f^{*}\left(\omega_{\pi}^{n}\right)=\omega^{n} \neq 0$ since $M$ is symplectic. This contradiction then shows that there are no fixed points.

REMARK 4.2. Note that the second proof above works for $M$ only being c-symplectic and $A$ being any action.

REMARK 4.3. Note also that the first proof uses the assumption of a nontrivial fixed set and geometry to create a class in the image of Hurewicz which violates the condition on $\omega$. The second proof recognizes that the condition on $\omega$ somehow reduces the problem to one involving the $K(\pi, 1)$ associated to $M$ and then uses what is known about this association when a circle acts to obtain a contradiction. It would be interesting if these proofs could be understood in a unified way.

\section{Extensions of the method}

5.1. Fibre number. The ideas presented above may be extended beyond the cases of symplectic and c-symplectic manifolds. To do this, we require a result of Gottlieb [G2]. Suppose $M \stackrel{i}{\rightarrow} E \stackrel{p}{\rightarrow} B$ is a fibration and $M$ is a space (such as a closed $N$-manifold) with $H^{r}(M ; \mathbb{Z})=0$, for $r>N$ and $H^{N}(M ; \mathbb{Z})=\mathbb{Z}$. Then $i^{*}: H^{N}(E ; \mathbb{Z}) \rightarrow H^{N}(M ; \mathbb{Z})$ has image $k \mathbb{Z}$ for some $k$. The number $k$ is called the fibre number of the fibration $p$ and is denoted by $\Phi(p)$.

THEOREM 5.1.1 [G2]. If the fibration is the Borel fibration associated to a smooth compact connected Lie group action on an orientable manifold, $M \rightarrow M G \rightarrow B G$, then the the fibre number, now denoted $\Phi(G, M)$, is nonzero if and only if some isotropy group contains a maximal torus of $G$. Thus, if $G$ is a torus $T^{k}$, then $\Phi\left(T^{k}, M\right) \neq 0$ if and only if there exist fixed points for the action.

Note that real coefficients suffice in the determination of the vanishing or nonvanishing of the fibre number.

5.2. A result of Yau. First, let us generalize a result of Yau by a very simple application of the $\lambda_{\hat{\alpha}}$-invariant. (Note that we only need an element $\hat{\alpha} \in \pi_{1}\left(M^{M}, 1_{M}\right)$, which may or may not come from a circle action, to obtain the theorem.) 
TheOREM 5.2.1. Let $M^{2 n}$ be a closed manifold and suppose $\hat{\alpha} \in \pi_{1}\left(M^{M}, 1_{M}\right)$. If there exist $x_{i} \in \operatorname{Ker}\left(\lambda_{\hat{\alpha}}: H^{2}(M) \rightarrow H^{1}(M)\right), i=1 \ldots n$ with $x_{1} \cdot x_{2} \cdots x_{n} \neq 0$, then the fibre number of the Wang fibration associated to $\hat{\alpha}$ is nonzero.

Proof. Let $M \stackrel{i}{\rightarrow} E \stackrel{p}{\rightarrow} S^{2}$ be the fibration induced from $\hat{\alpha} \in \pi_{1}\left(M^{M}, 1_{M}\right) \cong$ $\pi_{2}(\operatorname{Baut}(M))$. The Wang sequence associated to the fibration is

$$
\ldots \rightarrow H^{2}(E) \stackrel{i^{*}}{\rightarrow} H^{2}(M) \stackrel{\lambda_{\hat{\vartheta}}}{\rightarrow} H^{1}(M) \rightarrow \ldots
$$

and $\lambda_{\hat{\alpha}}\left(x_{j}\right)=0$ implies that there is some $\bar{x}_{j} \in H^{2}(E)$ with $i^{*}\left(\bar{x}_{j}\right)=x_{j}$. But this means that $i^{*}\left(\bar{x}_{1} \cdots \bar{x}_{n}\right)=x_{1} \cdots x_{n} \neq 0$. Hence, $\Phi(p) \neq 0$.

We now get two immediate corollaries of this approach. The first follows from the second part of Theorem 5.1.1 and Theorem 5.2.1. The second (Yau's result) is then a special case of the first (i.e. when $\operatorname{Ker}\left(\lambda_{\hat{\alpha}}: H^{2}(M) \rightarrow H^{1}(M)\right)$ is all of $\left.H^{2}(M)\right)$.

COROLlary 5.2.2. Suppose that $S^{1}$ acts effectively on $M^{2 n}$ and suppose $\hat{\alpha} \in$ $\pi_{1}\left(M^{M}, 1_{M}\right)$ is the element associated to the circle action. If there exist elements $x_{i} \in$ $\operatorname{Ker}\left(\lambda_{\hat{\alpha}}: H^{2}(M) \rightarrow H^{1}(M)\right), i=1 \ldots n$ with $x_{1} \cdot x_{2} \cdots x_{n} \neq 0$, then there are fixed points for the action.

P r o of. By Lemma 2.6 and the proof of Theorem 5.2.1, we see that there are elements $\tilde{x}_{k}$ with $j^{*}\left(\tilde{x}_{k}\right)=x_{k}$ for each $k$, where $j: M \rightarrow M S^{1}$ is the inclusion of $M$ in the total space of the Borel fibration. Hence, $\Phi\left(S^{1}, M\right) \neq 0$. By Theorem 5.1.1, there are fixed points.

Corollary 5.2.3 [Yau]. Suppose $S^{1}$ acts effectively on $M^{2 n}$ and $H^{1}(M)=0$. If there exist $x_{i} \in H^{2}(M), i=1 \ldots n$ with $x_{1} \cdot x_{2} \cdots x_{n} \neq 0$, then there exist fixed points for the action.

5.3. Actions on almost complex manifolds. The property that some product is nontrivial in the top dimension leads to other results which connect with symplectic topology. With this in mind, let $M^{2 n}$ be an almost complex manifold; that is, a manifold with an almost complex structure $J: T M \rightarrow T M$ (which, however, does not necessarily come from a symplectic structure). Suppose $S^{1}$ acts (smoothly) on $M$ so as to preserve the almost complex structure $J$. For a complex structure $J$ on a vector space $V$ of dimension $2 n, \mathrm{GL}(\mathbb{R}, 2 n)$ acts by $g \cdot J=g J g^{-1}$. If the structure is preserved, then $g \cdot J=J$, so $g J=J g$. For an almost complex structure $J$ on a manifold $M$ with $S^{1}$-action, the preservation of $J$ translates into the condition $g_{*} J=J g_{*}$ for each $g \in S^{1}$, where $g_{*}$ is the action induced on $T M$ from the action on $M$.

THEOREM 5.3.1. Suppose the circle acts on an almost complex manifold $\left(M^{2 n}, J\right)$ so as to preserve the almost complex structure. If $M$ has a nonzero Chern number, then the action has fixed points.

REMARK 5.3.2. Of course, this result applies to symplectic circle actions since, by Theorem 1.5, any symplectic circle action has an invariant almost complex structure in the homotopy class of structures determined by the symplectic form. Keeping this in mind, recall Theorem 2.10: if $c_{1}(M)=r \cdot \omega$ for $r>0$, then any symplectic $S^{1}$ action on $M$ is Hamiltonian. An important consequence of being Hamiltonian is the existence of 
fixed points. Theorem 5.3.1 also shows the existence of fixed points under the hypothesis $c_{1}(M)=r \cdot \omega$. Namely, since $\omega^{n} \neq 0$, the hypothesis provides a nonzero Chern number $c_{1}^{n}(M)[M]$. In fact, the approach to Theorem 5.3.1 given below also provides an alternative proof of Theorem 2.10. Namely, below we shall show that the hypothesis of $c_{1}(M)=r \cdot \omega$ implies the existence of an element $\tilde{\omega}=c_{1}(\theta) \in H^{2}\left(M S^{1}\right)$ as in Theorem 2.7. Hence, an $S^{1}$-action on such a symplectic manifold is Hamiltonian.

In order to prove Theorem 5.3.1, we employ an old trick which (at least) goes back to Borel-Hirzebruch [BoHi] and which has more recently been revived by Gottlieb [G2]. (In fact, Gottlieb alludes to a result analogous to Theorem 5.3.1 for complex manifolds and holomorphic actions.) The trick we refer to is the construction of the so-called (tangent) bundle along the fibres. Although the construction may be carried through in general, we shall confine ourselves to the following situation.

Let $M \stackrel{j}{\rightarrow} M S^{1} \rightarrow B S^{1}$ be the Borel fibration associated to a (smooth) circle action and let $T M$ denote $M$ 's tangent bundle. The circle acts on $T M$ as we have previously noted by derivative maps, so form

$$
\theta=\left(E S^{1} \times T M\right) / S^{1}=E S^{1} \times_{S^{1}} T M .
$$

Now, $M S^{1}=E S^{1} \times S^{1} M$, so clearly $\theta$ is a vector bundle over $M S^{1}$ and there is a commutative diagram

$$
\begin{array}{ccc}
T M & \stackrel{\epsilon}{\rightarrow} & \theta \\
\downarrow & & \downarrow \\
M & \stackrel{j}{\rightarrow} & M S^{1}
\end{array}
$$

of bundles where $\epsilon$ is the natural inclusion $(m, v) \mapsto\left[e_{0},(m, v)\right]$ for a chosen basepoint of $E S^{1}, e_{0}$. Also, note that we have

$$
\left.\theta\right|_{M}=j^{*} \theta=T M .
$$

The bundle $\theta$ is called the bundle along the fibres of the Borel fibration.

LEMMA 5.3.3. If $M$ has an $S^{1}$-invariant almost complex structure $J$, then the bundle along the fibres $\theta$ has a complex structure $\tilde{J}$ extending $J$ and $\epsilon$ is a mapping of complex vector bundles.

Proof. Define $\tilde{J}: \theta \rightarrow \theta$ by $\tilde{J}([e,(m, v)])=[e,(m, J v)]$, where $(m, v) \in T M$ and $e \in E S^{1}$. We must show this definition is well defined, so let $[e,(m, v)]=[f,(x, w)]$. This means that there is some $g \in S^{1}$ such that $\mathrm{eg}^{-1}=f, g \cdot m=x$ and $g_{*}(v)=w$. Then

$$
\begin{aligned}
\tilde{J}([f,(x, w)]) & =[f,(x, J w)]=\left[e g^{-1},\left(g \cdot m, J\left(g_{*}(v)\right)\right]=\left[e g^{-1},\left(g \cdot m, g_{*} J(v)\right)\right]\right. \\
& =[e,(m, J(v))]=\tilde{J}([e,(m, v)]
\end{aligned}
$$

where we have used the $S^{1}$-invariance of $J, J g_{*}=g_{*} J$, in the third line. Clearly, $\tilde{J}^{2}=-I$ since $J$ has this property and $\epsilon$ is a map of complex structures.

Proof of Theorem 5.3.1. Form the bundle along the fibres of the Borel fibration $\theta$ as above. From Lemma 5.3.3, we know that $T M$ is the pullback of the complex bundle $\theta$ by $j$. By the pullback property of Chern classes

$$
c(T M)=c\left(j^{*} \theta\right)=j^{*} c(\theta) .
$$


By hypothesis, some Chern number $c_{i_{1}}(M) \cdots c_{i_{k}}(M)[M] \neq 0$ (where $2 \sum_{s=1}^{k} i_{s}=2 n$ ). But then, in the top dimension of $M$, we have

$$
j^{*}\left(c_{i_{1}}(\theta) \cdots c_{i_{k}}(\theta)\right)[M]=c_{i_{1}}(M) \cdots c_{i_{k}}(M)[M] \neq 0
$$

so that the fibre number $\Phi\left(S^{1}, M\right)$ is nonzero. By Theorem 5.1.1, there exist fixed points.

\section{4. $S^{3}$-Actions on quaternion-Kähler manifolds}

Definition 5.4.1. A (closed) manifold $M^{4 n}$ is cohomologically quaternion-Kähler or $c$ - $Q K$ if there exists a class $\omega \in H^{4}(M)$ such that $\omega^{n} \neq 0$.

Manifolds fitting the description of Definition 5.4.1 are the topological analogue of the quaternion-Kähler manifolds of geometry. Recall that a manifold $M^{4 n}$ is quaternionKähler if $M$ has a metric such that there is a covering of $M$ by open sets $U_{i}$ which each have two almost complex structures $I$ and $J$ obeying:

i. $I$ and $J$ are isometries for the metric.

ii. $I J=-J I$. $I J$ is denoted by $K$ and the three almost complex structures $I, J$ and $K$ then represent the quaternions.

iii. The Levi-Civita derivatives of $I$ and $J$ are linear combinations of $I, J$ and $K$.

iv. For $x \in U_{i} \cap U_{j}$, the vector space of endomorphisms of $T_{x} M$ generated by the almost complex structures is the same for those structures associated to $U_{i}$ and $U_{j}$.

This definition is equivalent to saying that the holonomy group of $M$ is contained in $S p(n) S p(1)$. For more information on these manifolds, see [Bes] for example.

EXAMPLE 5.4.2. (1) Quaternionic projective space $\mathbb{H} P^{n}$ is a quaternion-Kähler manifold. Indeed, its holonomy coincides with its isotropy subgroup $S p(n) S p(1)$. Note that $\mathbb{H} P^{n}$ cannot have an almost complex structure for topological reasons [Mas], so that the $I$ 's, J's and $K$ 's given by the quaternion-Kähler structure can never be global.

(2) In [Kr] (also see [Bes, Proposition 14.92]), it was shown that a quaternion-Kähler manifold $M^{4 n}$ has a closed nondegenerate 4 -form $\Omega$ with $\Omega^{n} \neq 0$ and $[\Omega]=8 \pi p_{1}(E) \in$ $H^{4}(M)$, where $p_{1}(E)$ is the first Pontryagin class of the subbundle $E \subset \operatorname{End}(T M)$ determined by the $I, J$ and $K$ of $M$. Hence, a compact quaternion-Kähler manifold is c-QK.

(3) A closed c-symplectic manifold of dimension $4 n,\left(M^{4 n}, \omega\right)$ is c-QK since $\left(\omega^{2}\right)^{n} \neq 0$.

Now, for $\left(M^{4 n}, \omega\right)$ c-QK, suppose $A: S^{3} \times M \rightarrow M$ is a smooth action. The exponential law provides an element

$$
\hat{\alpha} \in \pi_{3}\left(M^{M}, 1_{M}\right) \cong \pi_{4}(\operatorname{Baut}(M))
$$

corresponding to the action $A$. As before, we have $S^{4} \rightarrow B S^{3} \rightarrow \operatorname{Baut}(M)$ and a consequent induced fibration

$$
M \stackrel{i}{\rightarrow} E \rightarrow S^{4}
$$

Again, as in the circle action case, we have a map of this fibration into the Borel fibration 
associated to the $S^{3}$-action,

$$
\begin{array}{ccccccc}
S^{3} & \rightarrow & M & \stackrel{j}{\rightarrow} & M S^{3} & \rightarrow & B S^{3} \\
\uparrow & & \| & & \uparrow \phi & & \uparrow \\
\Omega S^{4} & \rightarrow & M & \stackrel{i}{\rightarrow} & E & \rightarrow & S^{4} .
\end{array}
$$

Again we may consider $S^{4}$ as the 4-skeleton of the classifying space $B S^{3}$ so that the composition $S^{4} \rightarrow B S^{3} \rightarrow \operatorname{Baut}(M)$ is $\hat{\alpha}$. Indeed, since $H^{*}\left(B S^{3} ; \mathbb{Z}\right)$ is a polynomial algebra over $\mathbb{Z}$ on a degree 4 generator, the map $S^{4} \rightarrow B S^{3}$ induces an isomorphism in (integral) homology through degree 7 . By the Hurewicz theorem, we have $\pi_{i}\left(S^{4}\right) \cong$ $\pi_{i}\left(B S^{3}\right)$ for $i \leq 6$ and $\pi_{7}\left(S^{4}\right) \rightarrow \pi_{7}\left(B S^{3}\right)$ surjective. A diagram chase through the ladder of exact homotopy sequences

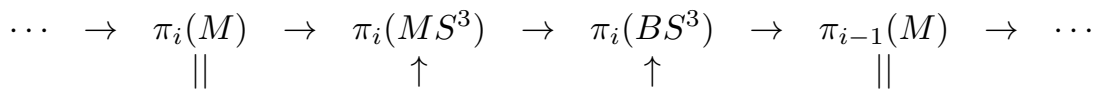

$$
\begin{aligned}
& \cdots \rightarrow \pi_{i}(M) \rightarrow \pi_{i}(E) \quad \rightarrow \quad \pi_{i}\left(S^{4}\right) \quad \rightarrow \quad \pi_{i-1}(M) \rightarrow \cdots .
\end{aligned}
$$

then shows that the map $\pi_{i}(E) \rightarrow \pi_{i}\left(M S^{3}\right)$ is an isomorphism for $i \leq 6$ and is surjective for $i=7$. Hence, again by Hurewicz,

LEMMA 5.4.3. $H_{i}(E) \cong H_{i}\left(M S^{3}\right)$ for $i \leq 6$ and $H^{i}(E) \cong H^{i}\left(M S^{3}\right)$ for $i \leq 6$.

Now, the Wang sequence for $M \rightarrow E \rightarrow S^{4}$ is

$$
\cdots \rightarrow H^{q}(E) \stackrel{i^{*}}{\rightarrow} H^{q}(M) \stackrel{\lambda_{\hat{q}}}{\rightarrow} H^{q-3}(M) \rightarrow H^{q+1}(E) \rightarrow \cdots
$$

with a derivation $\lambda_{\hat{\alpha}}$ as before. If $\left(M^{4 n}, \omega\right)$ is c-QK, then $\lambda_{\hat{\alpha}}(\omega) \in H^{1}(M)$ and we can make the

Definition 5.4.4. A smooth action $A: S^{3} \times M \rightarrow M$ on a c-QK manifold $\left(M^{4 n}, \omega\right)$ is $S^{3}$-c-Hamiltonian if $\lambda_{\hat{\alpha}}(\omega)=0$.

There is then an analogue to Proposition 2.3 and Theorem 2.7.

Proposition 5.4.5. An action $A: S^{3} \times M \rightarrow M$ on a c-QK manifold $\left(M^{4 n}, \omega\right)$ is $S^{3}$-c-Hamiltonian if and only if either of the following two conditions holds:

$$
\left\{\begin{array}{lll}
\exists & \bar{\omega} \in H^{4}(E) & \text { with } i^{*}(\bar{\omega})=\omega . \\
\exists & \tilde{\omega} \in H^{4}\left(M S^{3}\right) & \text { with } j^{*}(\tilde{\omega})=\omega .
\end{array}\right.
$$

DEFINITION 5.4.6. A maxtor point for a compact group action is a point for which the isotropy group at the point contains a maximal torus.

Of course a fixed point is always maxtor and maxtor means the same as fixed when the group acting is a torus, but what about other groups acting when we are not assured of fixed points? In [G2], it was shown that maxtor points always exist when $S^{3}$ acts on a manifold having $H_{i}(M ; \mathbb{Z})=0$ for $i=3 \bmod 4$. In a somewhat similar vein, we have

THEOREM 5.4.7. An $S^{3}$-c-Hamiltonian action on a c-QK manifold has maxtor points.

Proof. By Proposition 5.4.5, an $S^{3}$-c-Hamiltonian action $A: S^{3} \times M \rightarrow M$ on a c-QK manifold $\left(M^{4 n}, \omega\right)$ has $j^{*}(\tilde{\omega})=\omega$ for some $\tilde{\omega} \in H^{4}\left(M S^{3}\right)$. But (just as for the $S^{1}$ situation), $\omega^{n} \neq 0$, so $j^{*}\left(\tilde{\omega}^{n}\right)=\omega^{n} \neq 0$. Thus, the Borel fibration has a nonzero fibre number and we may apply Theorem 5.1.1. 
Questions and problems for the future 5.4.8. (1) The notion of $S^{3}$-c-Hamiltonian is not so clearly related to the usual notion of Hamiltonian as was c-Hamiltonian to Hamiltonian in the case of $S^{1}$. So, we may ask if there is a geometric notion of $S^{3}$-Hamiltonian in the case of actions on Quaternion-Kähler manifolds which is the geometric analogue of our topological idea. Does this geometric notion have properties similar to those of Hamiltonian actions on symplectic manifolds?

(2) With the first item in mind, the problem is to construct $S^{3}$-actions on QuaternionKähler manifolds.

(3) Finally, are there structure theorems for maxtor points analogous to those for fixed points?

\section{References}

[ABKLR] B. Aebischer, M. Borer, M. Kalin, Ch. Leuenberger and H. M. Reimann, Symplectic Geometry, Progress in Math., vol. 124, Birkhäuser, 1994.

[AL] M. Audin and J. Lafontaine, Holomorphic Curves in Symplectic Geometry, Progress in Math., vol. 117, Birkhäuser, 1994.

[AM] R. Abraham and J. Marsden, Foundations of Mechanics, Addison-Wesley, 2nd ed., 1978.

[AP] C. Allday and V. Puppe, Cohomological Methods in Transformation Groups, Cambridge Studies in Advanced Mathematics 32, Cambridge U. Press, 1993.

[Au1] M. Audin, The Topology of Torus Actions on Symplectic Manifolds, Progress in Math., vol. 93, Birkhäuser, 1991.

[Au2] M. Audin, Exemples de variétés presque complexes, L'Enseignement Math. 37 (1991), 175-190.

[BG] C. Benson and C. Gordon, Kähler and symplectic structures on nilmanifolds, Topology 27 (1988), 513-518.

[Bes] A. Besse, Einstein Manifolds, Ergebnisse der Math. und ihrer Grenz. (3), vol. 10, Springer-Verlag, 1987.

[BoHi] A. Borel and F. Hirzebruch, Characteristic classes and homogeneous spaces, Amer. J. Math. 80 (1958), 458-538.

[Br] G. Bredon, Introduction to Compact Transformation Groups, Academic Press, 1972 .

[BH] W. Browder and W-C. Hsiang, G-Actions and the Fundamental Group, Invent. Math. 65 (1982), 411-424.

[DGMS] P. Deligne, P. Griffiths, J. Morgan, and D. Sullivan, Real homotopy theory of Kähler manifolds, Invent. Math. 29 (1975), 245-274.

[Fr] T. Frankel, Fixed points and torsion on Kähler manifolds, Annals of Math. 70 no. 1 (1959), 1-8.

[G1] D. Gottlieb, Applications of bundle map theory, Trans. Amer. Math. Soc. 171 (1972), 23-50.

[G2] D. Gottlieb, The trace of an action and the degree of a map, Trans. Amer. Math. Soc. 293 (1986), 381-410.

[G3] D. Gottlieb, Lifting actions in fibrations, Lecture Notes in Math., vol. 657, Springer-Verlag, 1977, 217-254. 
[GM] P. Griffiths and J. Morgan, Rational Homotopy Theory and Differential Forms, Birkhäuser, 1981.

[Has] K. Hasegawa, Minimal models of nilmanifolds, Proc. Amer. Math. Soc. 106 (1989), 65-71.

[Has] A. Hattori and T. Yoshida, Lifting compact group actions in fiber bundles, Japan. J. Math. 2 (1976), 13-25.

[Kr] V. Kraines, Topology of quaternionic manifolds, Trans. Amer. Math. Soc. 122 (1966), 357-367.

[LO1] G. Lupton and J. Oprea, Symplectic manifolds and formality, J. Pure and Appl. Algebra 91 (1994), 193-207.

[LO2] G. Lupton and J. Oprea, Cohomologically symplectic spaces: toral actions and the Gottlieb group, Trans. Amer. Math. Soc. 347 no. 1 (1995), 261-288.

[Mas] W. Massey, The non-existence of almost complex structures on quaternionic projective spaces, Pac. J. Math. 12 (1962), 1379-1384.

[Mc1] D. McDuff, Symplectic diffeomorphisms and the flux homomorphism, Invent. Math. 77 (1984), 353-366.

[Mc2] D. McDuff, Examples of simply-connected, symplectic non-Kählerian manifolds, J. Diff. Geom. 20 (1984), 267-277.

[Mc3] D. McDuff, The moment map for circle actions on symplectic manifolds, J. Geom. Phys. 5 no. 2 (1988), 149-160.

[McS] D. McDuff and D. Salamon, Introduction to Symplectic Topology, Oxford Math. Monographs, Oxford U. Press, 1995.

[Mo] G. Mostow, Factor spaces of solvable groups, Annals of Math. 60 (1954), 1-27.

[No] K. Nomizu, On the cohomology of homogeneous spaces of nilpotent Lie groups, Annals of Math. 59 (1954), 531-538.

[On1] K. Ono, Equivariant projective imbedding theorem for symplectic manifolds, J. Fac. Sci. Univ. Tokyo IA Math. 35 (1988), 381-392.

[On2] K. Ono, Obstruction to circle group actions preserving symplectic structure, Hokkaido Math. J. 21 (1992), 99-102.

[On3] K. Ono, Some remarks on group actions in symplectic geometry, J. Fac. Sci. Univ. Tokyo 35 (1988), 431-437.

[Sp] E. Spanier, Algebraic Topology, McGraw-Hill, 1966.

[Su] D. Sullivan, Infinitesimal computations in topology, Publ. IHES 47 (1978), 269331.

[Th] W. Thurston, Some simple examples of symplectic manifolds, Proc. Amer. Math. Soc. 55 (1976), 467-468.

[Ti] D. Tischler, Closed 2-forms and an embedding theorem for symplectic manifolds, J. Diff. Geom. 12 (1977), 229-235.

[TO] A. Tralle and J. Oprea, Symplectic manifolds with no Kähler structure, Lecture Notes in Math., vol. 1661, Springer-Verlag, 1997.

[Wa] R. Warfield, Nilpotent Groups, Lecture Notes in Math., vol. 513, Springer-Verlag, 1976.

[Yau] S. T. Yau, Remarks on the group of isometries of a riemannian manifold, Topology 16 (1977), 239-247. 